\title{
Poder y dependencia: Los intermediarios locales en Siria Contemporánea
}

\author{
Power and dependence: Local intermediaries in contemporary Syria
}

Nour AL-HUSSEN VILLA

Universidad de Santiago de Compostela

alhussen.villa@gmail.com

https://orcid.org/0000-0001-6282-1064

Recibido 28/04/2021. Aceptado 15/10/2021

Para citar este artículo: Nour AL-HUSSEN VILLA (2021): "Poder y dependencia: Los intermediarios locales en Siria Contemporánea" en Revista de Estudios Internacionales Mediterráneos, 31, pp. 147-167.

Para acceder a este artículo: https://doi.org/10.15366/reim2021.31.008

\section{Resumen}

Durante décadas el régimen de los Asad ha extendido su dominio en las zonas periféricas del país a través de la articulación de una red de intermediarios de diferente naturaleza. Estos agentes locales eran encargados de asegurar la estabilidad y ejercer la contención de posibles sublevaciones a cambio de privilegios. La implosión de las estructuras del Estado el conflicto podría haber supuesto una oportunidad para terminar con las prácticas clientelistas del orden social preestablecido. No obstante, los resultados obtenidos en este documento demuestran no solo la supervivencia de la vieja estructura de intermediarios, sino también la emergencia de una nueva.

Palabras clave: Siria, intermediarios, conflicto, clientelismo, gobierno local.

\section{Abstract}

During decades the Asad regime has extended its domain in peripheral areas of the country through the establishment of a network of intermediaries of different origins. The mission of these local 
agents was to ensure the stability and to contain possible uprisings in exchange of privileges. The implosion of the state structures during the conflict could have supposed the opportunity to break with the clientelism practices of the prearranged social order. However, the results obtained in this document show how not only have the old structure of intermediaries survived, but also the emergence of a new one.

Key words: Syria, intermediaries, conflict, clientelism, local government.

\section{Introducción}

Transcurridos diez años desde el inicio de la crisis siria, sus efectos multidimensionales deberían hacernos repensar la forma de entender y abordar las problemáticas del país levantino. El estallido del conflicto, precedido por el levantamiento popular protagonizado en 2011 no solo ha causado la muerte de más de 511.000 civiles (Human Rights Watch [HRW], 2019), el desplazamiento forzoso de otros 12 millones (United Nations Office for the Coordination of Humanitarian Affairs [UNOCHA], 2019) y una destrucción material generalizada, sino también la alteración de los cimientos que sostenían la arquitectura de la dictadura asadiana ${ }^{1}$ vigente desde hace más de cuatro décadas.

Pese a que su surgimiento fue influenciado por las protestas que tuvieron lugar en el Norte de África entre fines del año 2010 e inicios de 2011, el levantamiento popular sirio tenía un fundamento propio. Además de la petición de la reforma del régimen, la libertad de los presos políticos, la creación de un sistema multipartidista o la derogación de las leyes de emergencia (Álvarez-Ossorio, 2016), el movimiento inicialmente pacífico también exigía el fin de la corrupción y la desigualdad junto al establecimiento de un Estado de bienestar, lo que implicó la ruptura de un contrato social autoritario establecido hasta entonces (Ruiz de Elvira y Zintl, 2014).

No obstante, determinados objetos de estudio han monopolizado el debate en torno a la crisis siria. El refuerzo de los estándares de seguridad globales, tras acontecimientos clave de nuestro siglo como es el caso de los atentados del $11 \mathrm{~S}$, ha obligado a poner el foco de análisis sobre temáticas de índole securitaria tales como la crisis migratoria o el ascenso del yihadismo. Asimismo, las consecuencias de la implicación de potencias mundiales en el conflicto con sus respectivas alianzas y fricciones favorecieron la producción de análisis que abordan el conflicto desde una perspectiva meramente geoestratégica.

De esta manera, los estudios publicados sobre las dinámicas internas preestablecidas y/o generadas durante el conflicto continúan siendo minoritarios. Por lo contrario, y debido a su alto grado de internacionalización, se ha puesto más el acento en las consecuencias globales del conflicto, que en sus verdaderas causas políticas o económicas. Por ejemplo, se suele ignorar las graves condiciones socioeconómicas existentes en Siria antes del levantamiento popular: el crecimiento de la población junto al aumento de la tasa de paro, y un reparto desigual de la riqueza desembocó en el empobrecimiento de la población de $30.01 \%$ en 2004 a 33.6\% en 2007 a pesar del incremento continuo del PIB. Asimismo, las prácticas corruptas tanto dentro del marco institucional como a pie de calle por parte de las autoridades se hicieron más visibles bajo el mandato de Bashar Al Asad, hasta el punto de situar al país en el año 2008 en el puesto 147 en el Índice de Percepción de la

\footnotetext{
${ }^{1}$ Término utilizado para hacer referencia a "la Siria de los Asad". Es un término recurrente por ejemplo en los trabajos de Santiago Alba Rico y en artículos del blog Traducciones de la Revolución Siria, disponible en:

http://traduccionsiria.blogspot.com [consulta: 13/11/2020].
} 
Corrupción elaborado por ONG Transparency International, que incluye un total de 180 países (Ruiz de Elvira, 2012).

En tal caso, el debate académico minoritario enmarcado en la dimensión interna del conflicto y alejado de la perspectiva securitaria o geoestratégica, se habría centrado más en las reformas político-institucionales para una transición democrática o en los esfuerzos necesarios para alcanzar una estabilización que permitiera el paso a una fase de postconflicto. Sin embargo, aspectos como la redistribución de la riqueza y la relación de poder entre el nivel local y el nivel nacional son marginados, no solo en los textos académicos que abordan la crisis siria, sino también en los existentes sobre Oriente Medio y Norte de África en general (Ruiz de Elvira et al, 2019: 2-7).

Por ello, nos parece interesante señalar que una de las claves inexploradas para explicar la fortaleza y supervivencia del régimen de los Asad se centra en habilidad de haber generado redes informales de contactos y el establecimiento relaciones personales, o lo que Nikolaos van Dam denomina "power elite" (van Dam, 2011). La finalidad de estas redes fue ejercer como instrumento de contención y control, así como reafirmar la autoridad y asegurar la permanencia del régimen en el poder. Este vínculo centro - periferia fue forjado generalmente entre Damasco y sus agencias de seguridad con lo que denominaremos actores intermediarios a lo largo de este documento: notables, jefes de tribus, cabezas de familias que históricamente contaban con una fuerte influencia, líderes religiosos, así como funcionarios de rango medio.

Se puede afirmar que la ausencia de una perspectiva local que aborde estas dinámicas informales, esenciales para comprender el funcionamiento de la Siria contemporánea y la Siria post-2011, contribuye a que se nos presente una panorámica completa del de la configuración del país levantino. Es por ello que el siguiente artículo tiene como objetivo analizar el modelo de control político imperante en la Siria de los Asad basado en las relaciones informales y redes de dependencia.

El siguiente documento trata de responder a las siguientes preguntas ¿Cuál es el origen de este sistema de control? ¿Cómo se han ido articulando estas relaciones informales en las diferentes fases del mandato del clan de los Asad? ¿Ha originado el levantamiento popular y el conflicto un nuevo orden sociopolítico? ¿En qué medida ha transformado el conflicto las relaciones de dependencia establecidas bajo el mandato de los Asad?

\section{Intermediarios: conceptualización y breve revisión a la literatura académica}

\section{¿Qué es un intermediario?}

En este documento hemos elegido utilizar el término de "intermediarios", también denominados por otros académicos como "fixers" (Diwan et al, 2019; Derderian et al, 2016) para hacer referencia a aquellos individuos que en el contexto sirio han ejercido de nexo entre las comunidades locales $y$ 
la autoridad central. Tomamos como referencia para la denominación de intermediarios, así como para su definición general, el último documento publicado en relación con nuestro objeto de estudio: Local intermediaries in post-2011 Syria, de Khedder Khadour y Kevin Mazur (Khaddour y Mazur, 2019)2. Con "comunidad local nos referimos a un grupo de personas unidas por lazos sociales y familiares y que están en constante interacción, que habitan en un espacio determinado compartiendo valores, experiencias e intereses.

Cuando hablamos de autoridad central, hacemos alusión a actores ajenos a la comunidad local que poseen autoridad política, proveen recursos, generan necesidades y ejercen el control de manera sistemática para mantener el orden. En el caso sirio pre-2011 identificaremos a esta "autoridad central" con el Estado sirio. No obstante, tras el estallido del conflicto, la pérdida del monopolio de la violencia y la implicación de otros actores han diversificado y re-dirigido el centro de poder, causando que actores estatales así como agencias y organizaciones internacionales, tengan la oportunidad de transformar la antigua estructura de poder y dependencia, o incluso impulsar la creación de una nueva.

Lejos de tratarse de una mediación desinteresada, o el mero ejercicio como canal de transmisión de información entre las partes, podemos señalar esta relación como jerárquica y vertical, basada en transacciones materiales e inmateriales, lo que enmarca a Siria en un sistema neopatrimonial. A cambio de su contribución a perpetuar la dominación política del régimen en las localidades y áreas periféricas del país, facilitar negociaciones en beneficio del orden político establecido y de las estrategias personales de los aparatos de seguridad, así como movilizar los recursos oportunos, los intermediarios obtienen ganancias económicas o privilegios políticos y sociales como premio a su lealtad transaccional ${ }^{3}$.

Al mismo tiempo, esta actividad ejercida de forma sistemática refuerza su posición social dentro de la comunidad local como interlocutores y legítimos representantes de los intereses generales. Podemos afirmar que el poder de los intermediarios se basa en dos factores esenciales: la capacidad de influencia y el acceso a los recursos. En referencia a esto último, en el contexto sirio podemos diferenciar cuatro ámbitos: el acceso a la estructura de toma de decisiones, así como a los recursos económicos (petróleo, gas, control arbitrario sobre la moneda, licencias), la capacidad de institucionalizar relaciones sociales, y el acceso a la esfera público y a garantizar derechos políticos (Becker, 2006: 69-91).

\section{Breve revisión a la literatura académica sobre la figura de los intermediarios en el contexto sirio}

Siendo una temática minoritaria abordada en la literatura existente sobre Siria, algunos autores han contribuido invertido sus esfuerzos en abarcar el fenómeno de los intermediarios en sus estudios, y, por lo tanto, ayudar a la comprensión de las dinámicas informales de distribución de poder en Siria. Podemos diferenciar dos grupos: los que han abordado la temática antes del estallido del conflicto, y los que han producido sus análisis tras el comienzo de este.

Durante la década de los sesenta, se publicaron dos obras de gran impacto sobre nuestro objeto de estudio. Ambas analizan la dominación de grupos de notables de zonas urbanas y rurales en

\footnotetext{
2 No obstante, mientras que ellos definen a los intermediarios como enlace entre las comunidades locales y las autoridades externas, diferimos en la conceptualización de este segundo término, ya que resulta confuso.

${ }^{3}$ Con este término queremos hacer alusión a una lealtad sustentada únicamente por los privilegios y beneficios obtenidos en su práctica.
} 
diferentes períodos históricos, se trata de Muslim Cities in the Later Middle Ages (Lapidus, 1967) y Ottoman Reform and the Politics of Notable (Hourani, 1968). Especialmente debido a su gran impacto, este segundo trabajo es un punto de partida fundamental para analizar cómo la creación de esta red de intermediarios constituyó un elemento central del sistema de gobierno del Imperio Otomano, ya sea antes o después de la implementación de sus reformas denominadas "Tanzimat". En su obra el autor afirma que las políticas urbanas de las provincias otomanas no pueden ser analizadas sin abordar las "políticas de notables", o lo que en términos de Max Weber constituye una dominación patriarcal o patrimonial sustentada por élites (Hourani, 1968: 41).

El trabajo de Hourani fue presentado en una conferencia en la Universidad de Chicago en 1966, para ser publicado dos años después en un volumen editado por William Polk y Richard Chambers. No obstante, a pesar de considerarse una referencia principal, este trabajo también suscitó diversas críticas ya que su noción de sistema de patronazgo está influenciada por la teoría del funcionalismo estructuralista, que se encontraba en pleno apogeo en el momento de su elaboración. Para los críticos de Hourani y en líneas generales, su visión funcionalista es anacrónica y reduccionista (Salzmann, 2004: 17), ya que entiende las relaciones y dinámicas de los intermediarios como interacciones estables y monolíticas, destinadas a asegurar la estabilidad dentro de una sociedad que podría parecer libre de conflictos internos (Gelvin, 2006: 19-29).

Por su parte, Hannah Batatu también realizó una contribución valiosa con su obra Syria's Peasantry, The Descendents of its Lesser Rural Notables, and Their Politics (Batatu, 1999) que nos permite desde una perspectiva de clase aproximarnos a las estructuras de poder del partido al Baaz afianzadas tras el golpe de Estado del 1963. Este exhaustivo estudio sociopolítico sobre la Siria contemporánea alberga un compendio de entrevistas realizadas a agricultores y líderes políticos que arrojan luz sobre los orígenes rurales de la cúpula partido Al Baaz y cómo esta ha liderado una transformación ocupando el epicentro del poder monopolizado hasta entonces por líderes de determinados orígenes y clases sociales. Otra revisión interesante sobre la configuración de la dominación local en siria la podemos encontrar en forma de cuatro estudios de caso en el trabajo de Raymond Hinnebusch titulado Local Politics in Syria: Organization and Mobilization in Four Villages Cases (Hinnebusch, 1976).

De manera más específica, el trabajo de Dawn Chatty ha estado centrado en el estudio de la evolución de los beduinos en el mundo árabe. En el contexto sirio, la autora analiza el papel de las tribus como intermediario entre sus miembros y sus redes de solidaridad y los aparatos de seguridad del Estado. En su trabajo The Bedouin in Contemporary Syria: The Persistence of Tribal Authority and Control (Chatty, 2010: 24-49) analiza la relación de clientelismo y patronazgo establecida entre líderes de tribus y miembros del régimen ${ }^{4}$. En parte, este objeto de estudio es compartido por el investigador Haian Dukhan, cuya tesis doctoral se ha centrado en el estudio de las tribus sirias y su relación con las autoridades centrales desde la caída del Imperio Otomano hasta el levantamiento popular de 2011. El resultado ha dado lugar su primer libro State and Tribes in Syria: Informal Alliances and Conflict Patterns (Dukhan, 2019).

\footnotetext{
${ }^{4}$ Se recomienda también la lectura de la entrevista realizada a la autora por Aron Lund para Carnegie Middle East Center. Disponible en: https://carnegie-mec.org/diwan/60264 [consulta: 7/12/2021].
}

REIM № 31 (diciembre 2021) ISSN: $1887-4460$ 
Finalmente, entre las obras más recientes y significativas nos encontramos la ya mencionada Local Intermediaries in post-2011 Syria: Transformation and Continuity (Khaddour y Mazur, 2019). Los editores dirigieron cinco investigaciones llevadas a cabo por investigadores juniors en diferentes localidades sirias con el fin de analizar el rol de varios intermediarios durante el levantamiento popular y posteriormente durante el conflicto.

\section{Contexto histórico y origen de los intermediarios: Los ayan en el Imperio Otomano}

Entre 1760 y 1860, período pre-Tanzimat no existía un vínculo directo entre el Sultán y sus súbditos fuera de la capital imperial. La única manera de extender el control desde el centro del imperio y ejercer una dominación eficiente, especialmente en las provincias musulmanas, era a través de la mediación de personalidades de relevancia social y con poder de influencia. El conocimiento de estos individuos y familias sobre el contexto local facilitaba un control más eficaz que si lo ejercieran otros individuos ajenos a la cultura, a las dinámicas de relación social y a las costumbres propias de las provincias árabe-musulmanas. Es el caso de los gobernadores y oficiales designados desde Estambul, que en ocasiones no eran conocedores de la lengua ni de las posibles estrategias compatibles con las prácticas locales para el mantenimiento del orden. De esta manera, se constituía una relación centro-provincia basada en el equilibrio de poder y en la dependencia mutua, ya que estas personalidades locales denominadas como "Ayans" ${ }^{\prime 5}$ poseían la capacidad oficial de representar los intereses generales de sus localidades frente al sultán y a su vez, ejercer como una extensión de la autoridad de este. Es así como se configura la base de la política urbana del Imperio Otomano que Albert Hourani denomina como "Política de Notables" y a la que ya hemos hecho referencia anteriormente.

Albert Hourani distingue tres grupos diferentes con capacidad de ejercer de varias maneras este poder social en las provincias otomanas. Por un lado los ulema, cuyo poder emana de su pertenencia a familias reputadas y de su posición religiosa, por otro lado los jefes de guarniciones militares locales, cuya fuente de poder se basa en su autoridad militar y en el control inmediato del ejército, y por último los llamados "notables seculares" o individuos cuyo poder proviene de una tradición política o militar, la memoria de un ancestro, la solidaridad social con otro grupo equivalente "assabiyah", o en el control de la producción agrícola.

Independientemente de cuál fuera la razón en la que se basa su status (autoridad religiosa, militar, social o política), el intermediario era consciente de la ventaja que suponía el ejercicio de su rol. Su posición de liderazgo emanaba del poder que le dotaba el acceso a la autoridad y a los recursos, y al mismo tiempo, su posición de líder le concedía una diferenciación sobre el resto de los miembros de la comunidad, lo que facilitaba su conexión con la autoridad central y convertirse en una pieza indispensable para el ejercicio del control imperial. De esta manera, la relación de dependencia también era establecida con el resto de la población en un sentido vertical.

En este sentido, tanto el comportamiento como las relaciones personales en la sociedad otomana se regían por el concepto del "Had" (límite en turco y árabe), determinado por la pertenencia a una familia, posición, religión, clase y la posesión de riqueza. De acuerdo con el "Had”, la clase dirigente

\footnotetext{
${ }^{5}$ Aunque sea el término más utilizado, existe un amplio abanico de denominaciones que podemos encontrar en la literatura: agha, amir, derebey, mutegalibe, son algunas de ellas. La diversidad de términos se debe a la existencia de varias fuentes de las que emana la autoridad del notable. A pesar de existir un consenso sobre el uso del término ayan, Albert Hourani utiliza este para referirse específicamente a los notables seculares.
} 
posee su honor "sharif" y este constituye un valor intocable, ya que se trata de un indicador de su status. De hecho, el concepto del "Had" contribuyó de forma sustancial a la consolidación de una dinámica de abuso de poder consentida, ya que según su doctrina cada persona con poder poseía el derecho de obrar con total autonomía en su ámbito más cercano, siempre y cuando fuera conforme a los dictados de la tradición. Esto implicaba la sumisión total de los que se encontraban en una escala inferior, y su compromiso a aumentar la riqueza de los más poderosos y la aceptación sin condiciones de sus órdenes, siempre y cuando estos ofrecieran a cambio su protección. Esta práctica o acuerdo tácito era denominada como "intisap" (Shaw y Shaw, 1977: 166).

Para Eugene Rogan "La naturaleza de la dominación local difería de una región a otra" (Rogan, 2010: 76), de la misma manera que el grado de peligrosidad que implicaba la emergencia de los caudillos locales para la autoridad de Estambul variaba en función de su proximidad geográfica del centro del Imperio. Esta amenaza venía determinada por el alto grado de autonomía del que gozaban estas figuras y la libertad con la que operaban al margen del control imperial. En este sentido, se explica también la ambigüedad en su posicionamiento a hora de obrar en busca de sus intereses, pero sin llegar a contradecir los mandatos del Imperio, ya que de esa lealtad (al menos aparente) dependía su acceso a los recursos. Además, con el fin de afianzar su posición y crear redes, también era frecuente recurrir a la estrategia de crear coaliciones con otras familias o líderes, con el objetivo de intensificar el ejercicio de su poder o alcanzar intereses comunes. Estas relaciones horizontales eran generadas principalmente gracias a los matrimonios concertados entre miembros de familias de distintos rangos y tipos de notables, o también a través del establecimiento de acuerdos con comerciantes de renombre.

Podemos centrarnos geográficamente en Damasco, que constituye un ejemplo claro sobre las relaciones centro-provincia establecidas durante el Imperio Otomano, ya que la capital actual de Siria conformaba un enclave de vital importancia para la Sublime Puerta, en especial por su ubicación en la ruta de peregrinación. En 1707 Estambul designa como gobernador a un miembro de una familia poderosa y con múltiples conexiones locales, Al Azm, que a día de hoy todavía mantiene influencia en política damascena y siria (Quataert, 2005: 103). Lejos de suponer una mera designación, el poder otorgado a la familia Azm desde Estambul y su reconocimiento como élite, deja entrever la importancia del rol de las familias locales para el centro imperial.

Es durante el siglo XVIII bajo los mandatos del Sultán Selim III (1789-1807) y especialmente el de Mahmut II (1808-1839) cuando el Imperio Otomano emprende las primeras medidas modernizadoras a través de la implementación sucesivos programas con el objetivo de centralizar el poder y controlar de forma más exhaustiva la vida diaria en las provincias, concediendo mayor poder a los notables y restándoselo a los gobernadores en la segunda mitad del siglo (Emrence, 2008: 294). Esta acción no solo se enmarcó dentro de una estrategia política, sino también en una financiera, ya que buscaba hacer frente a las presiones internacionales y mitigar las graves consecuencias de la frágil situación económica que atravesaba el Imperio por aquel entonces. Esta medida también buscaba frenar el abuso de los gobernadores y oficiales provinciales, ya que implicaba la delegación de la responsabilidad de recaudar impuestos a los intermediarios locales a cambio de pagos por adelantado. Al mismo tiempo, esta competencia supone un privilegio que requiere tanto de conexiones políticas en todos los niveles, así como la creación de una cadena de favores e intereses entre más cinco mil personas (Emrence, 2008: 293). 
Este período de primeras reformas culminó con la aplicación de las llamadas Tanzimat (1839-1876), que crearon un gobierno centralizado y liderado por una nueva clase dirigente formada por una moderna generación de otomanos dispuestos a hacer frente a la crisis. El objetivo principal de las reformas era extender el control, llevando a cabo la reproducción exacta de la estructura administrativa central a pequeña escala en las provincias (siempre con la supervisión y el control de Estambul). Asimismo, se llevan a cabo varias medidas importantes: la reformulación del sistema de impuestos, la reestructuración de las divisiones administrativas con el fin de establecer un equilibrio entre población y riqueza, y por último, la reducción de la autonomía de los gobernadores y otros oficiales, mediante la creación de consejos compuestos por representantes de la clase dirigente y de la élite local. Es por ello que a efectos prácticos el poder e influencia de los notables no se vio afectado por las nuevas reformas, aunque estas buscaran la igualdad entre los súbditos. Podemos afirmar entonces que las reformas facilitaron la institucionalización y la inclusión dentro de la administración de estos individuos gracias a la riqueza e influencia que habían acumulado en las décadas anteriores (Inalcik, 1976: 13).

Tal y como hicieron los otomanos, los franceses también quisieron establecer un orden político basado en la interacción y negociación con socios locales durante su mandato en Siria (1920-1946). A diferencia de sus sucesores, los franceses carecían de la legitimidad inherente del Sultán en su día, por lo que la procura de la estabilidad en la región suponía su objetivo para mantener su mandato que podemos calificar como un dominio indirecto (Khoury, 1991). Un ejemplo claro de ello fue la relación que establecieron con las tribus. Los franceses trataron de lidiar con los beduinos de forma pragmática, ya que estos podían constituir una fuente de conflicto e inestabilidad, no solo porque habían tomado parte del levantamiento nacionalista que tuvo lugar entre los años 1925-1927, sino también porque eran frecuentes las fuertes disputas protagonizadas entre las propias tribus. Por ello, los franceses mantuvieron algunos acuerdos establecidos en la era otomana, y adecuaron otros a sus intereses más inmediatos. Por ejemplo, terrenos de gran superficie fueron registrados a nombre de líderes concretos, así como algunas leyes fueron adaptadas específicamente para las tribus. Por supuesto, la dinámica de dependencia y de intercambio de intereses continuó generándose de manera muy similar en esta etapa. Algunos líderes locales siguieron considerando el ejercicio de control entre sus comunidades y el mantenimiento de la estabilidad en sus zonas como una manera de generar beneficios personales y económicos. Es el caso por ejemplo de la tribu de Ruwalla, que negoció con las autoridades francesas proteger el estrecho el tramo sirio de Tapline $^{6}$. Asimismo, el mandato francés otorgó nueve asientos en el parlamento a los líderes de las tribus más poderosas (Chatty, 2010: 29-49).

\section{Los intermediarios en la era de Al Baaz: Del corporativisimo autoritario al capitalismo clientelar}

Desde su ascenso en 1963, y siendo consciente de la cultura clientelar practicada en etapas anteriores, el Partido Baaz sirio generó una estructura de poder formada por redes formales e informales, articuladas por identidades subnacionales: secta, religión, etnicidad, y tribu que le permitió integrar, promover o marginar a grupos según sus intereses. Es por ello que la gestión de esta estructura ha estado caracterizada por el trato desigual y el reparto de recursos y privilegios dependiendo de la lealtad demostrada por estos grupos y de su eficacia a la hora de ayudar al régimen a mantenerse en el poder y contener a la disidencia. Este elemento de desigualdad resulta clave para comprender la articulación del poder a nivel local, ya sea su aplicación a nivel geográfico

\footnotetext{
${ }^{6}$ Se trata de un oleoducto ahora desaparecido que unía el golfo pérsico con el mediterráneo.
} 
(por ejemplo, las zonas del noroeste tradicionalmente más marginadas que la costa) o a nivel sectario (el trato de favor rotatorio entre alauitas y sunnitas). Según Heiko Wimmen, por este mismo motivo podemos señalar a Siria como un "sistema de regiones" ya que más que un proceso de Estado-Nación, o Estado territorial lo que ha desarrollado es un modelo de Estado de territorios, en el que el régimen ha tenido que estar en constante negociación con la población local (Wimmen, 2016: 1-17). Podemos diferenciar tres fases de evolución en la distribución de poder a través de redes formales e informales en la Siria baazista pre-conflicto: la fase pre asadiana, la fase bajo dominio de Hafez Al Asad y, por último, la fase bajo el mandato de Bashar Al Asad.

Como contexto previo, podemos señalar que, durante la década de los sesenta, el partido Baaz extendió su dominio y su poder concentrado hasta el momento en la capital, irrumpiendo en las localidades a través de la creación de una nueva élite afín al régimen. Esta cantera estaba formada principalmente por jóvenes con formación superior, diseñada explícitamente para sustituir a las élites tradicionales heredadas del período otomano. La principal estrategia del partido durante la década de los sesenta fue agrupar a esas nuevas élites mediante la creación de organizaciones de masa y colectivos bajo su paraguas, como fue por ejemplo el caso del Sindicato Agrícola, o la cooptación de otros ya existentes como la Cámara de Comercio. En este sentido Volker Perthes describe esta estrategia de articulación, absorción, y unificación de las élites dentro de los organismos del partido dominante como un sistema de "corporativismo autoritario" muy similar a los modelos que podemos encontrar en América Latina durante los setenta y ochenta (Wimmen, 2016: 10), mientras que Hinnebusch opta por denominarla como "corporativismo populista" (Hinnebusch, 1976: 565). No obstante, la creación de estas nuevas élites no consiguió minar la capacidad de influencia de las élites tradicionales, produciéndose así una coexistencia entre ambas.

\section{El ascenso de Hafez Al Asad al poder: Cooptación y asignación de roles diferenciados para los intermediarios locales (1971-2000)}

Durante los años setenta y ochenta podemos observar como los intermediarios locales son aglutinados de manera oficial bajo el paraguas del régimen. En un contexto convulso que atravesaba siria durante esas dos décadas con acontecimientos como pueden ser la guerra con Israel en 1973, la represión ejercida en contra de los Hermanos Musulmanes entre 1978 y 1982, y la implicación en la guerra civil libanesa entre 1970 y 1980, los intermediarios resultaron esenciales para la contención y estabilización de las localidades.

En 1971 Hafez Al Asad es proclamado presidente ${ }^{7}$, y centra su objetivo en continuar extendiendo el control desde la capital a las localidades a través de los intermediarios locales, pero con una visión diferente de la que hasta entonces había puesto en práctica su partido. Lejos de crear una competición entre la nueva élite creada y las élites locales tradiciones, busca absorber formalmente a ambas dentro de las estructuras del Estado. Es en 1983, un año posterior a la revuelta de los Hermanos Musulmanes, se publica la nueva Ley de Administración Local. El objetivo de esta ley es

${ }^{7}$ Es importante señalar que en la literatura es obviado el hecho que a pesar de que el nombramiento oficial de Hafez al Asad fue en el 1971, su ascenso al poder fue previo con el golpe de Estado que lideró desde su posición como ministro de defensa. 
afianzar en un marco legal, la creación de cuerpos y consejos en los que se consigue articular a las élites locales como funcionarios o intermediarios que ejercen de nexo entre Damasco y la localidad (Khaddour, 2017: 5). La asignación de puestos oficiales (gobernadores, jefes de consejos provinciales, jefes de districtos) se convirtió en un canal esencial para contener y cooptar a las élites locales, ofreciéndoles privilegios pero sin una capacidad de decisión política (Khaddour, 2017: 5).

Cabe señalar que tras la represión ejercida a los Hermanos Musulmanes, en la década de los ochenta se comienza a configurar la fórmula con la que el régimen ha asegurado su éxito. La paradoja de poder que sustentó al régimen se basaba en que "a pesar de su ideología secular, ha tratado de cooptar el discurso religioso como un medio para compensar la fragilidad de sus respaldos populares" (ICG, 2004: 16). De esta manera, la imagen de régimen laico y de izquierdas que reprimía a un colectivo sunní debía de ser compensada de alguna manera ${ }^{8}$, una de ellas fue el nombramiento de clérigos que ayudaran al proceso de contención de la base social ${ }^{9}$. Asimismo, "Pese a que el régimen sirio nunca ha intentado producir clérigos baazistas, tampoco ha escatimado esfuerzos a la hora de cooptar a las cabezas visibles de las influyentes cofradías sufíes" (Álvarez-Ossorio, 2011: 173). También podemos observar que además del nombramiento de clérigos "desde la masacre de Hama, se ha publicitado un islam moderado y apolítico mediante la construcción de mezquitas (8.000 hoy en día), 120 escuelas al-Asad para el aprendizaje del Corán, 22 institutos superiores de ciencias religiosas y cerca de 300 (de un total de 600) asociaciones civiles islamistas" (ÁlvarezOssorio, 2011: 168). De esta manera, la carta sectaria fue aplicada durante estos años con el fin de aglutinar a las minorías (cristianos, ismaelitas y drusos) y dividir a la mayoría sunní (que representa el $80 \%$ de la población siria) para minimizar su potencial poder político (Balanche, 2009).

Otro ejemplo de la supervivencia de los intermediarios locales a lo largo de los años fue la visita del historiador Hanna Batatu a la aldea de Ibbin Samaan en 1985. Durante su estancia fue testigo de cómo las familias terratenientes que habían sido influyentes durante el período baathista continuaban manteniendo su poder e incluso tenían el derecho de recolectar un porcentaje de las tasas aplicadas a los agricultores (Batatu, 1999).

Durante este período temporal (1971-1990) es importante diferenciar el rol y los rasgos característicos de las élites locales "wasata mahalliyin" y los miembros representantes del régimen "shabakat al nizam" que operaban de forma paralela a la administración local oficial. Por una parte, las élites locales estaban conformadas generalmente por miembros de familias prominentes. Estas mostraban un compromiso férreo con el sentido de la solidaridad y la justicia, así como buscaban mantener su honor y su reconocimiento social. No obstante, a pesar de obtener un beneficio por desempeñar su papel intermediario, tenían como objetivo representar el interés colectivo de su comunidad. Por otra parte, los oficiales del régimen, que frecuentemente incluían a miembros del aparato de inteligencia "mujabarat" podían ser originarios de la localidad o no, poseían mayor capacidad de decisión y sus objetivos estaban más centrados en sus propios intereses personales que en los intereses de la comunidad local (Khaddour, 2017: 4-6). Esta estructura se mantuvo casi intacta hasta la muerte de Hafez Al Asad, mientras se gestaba por un lado la preparación de Bashar Al Asad como heredero a la presidencia ${ }^{10}$, y por otro lado, un proceso de liberalización económica que alcanzaría su máximo esplendor en el 2000 con el inicio del mandato de este.

\footnotetext{
${ }^{8}$ Para mayor profundidad en este proceso, se recomienda la lectura de PIERRET, Thomas (2009): "Sunni Clergy Politics in the Cities of Ba'thi Syria", en Lawson, Fred (dir.): Demystifying Syria, Londres, Saqi.

${ }^{9}$ Entrevista a Yassin Swehat, escritor y editor de Al-Jumhuriya (15/10/2021).

${ }^{10}$ En principio, el heredero era su hijo Basel. No obstante este falleció en un accidente y el sucesor pasó a ser Bashar Al Asad.
} 


\section{El mandato de Bashar Al Asad: Las reformas neoliberales y la emergencia de una nueva élite}

En el año 2000 Bashar Al Asad asume el poder, convirtiendo a Siria en la primera república hereditaria de Oriente Medio. La liberalización de la economía, una de sus primeras decisiones en el poder, repercutió de forma directa en las redes formales e informales cultivadas y consolidadas desde hacía décadas, y por lo tanto en la manera de gestionar la relación centro-periferia. Por ejemplo, en diciembre de 2000 se llevó a cabo la privatización de las granjas estatales, la mayoría de ellas ubicadas en el noroeste sirio. Esta decisión visibilizó la intención de beneficiar a las élites y con ello, despertó el resentimiento entre la población, ya que la redistribución beneficiaba a tribus concretas y a los grandes terratenientes leales al régimen (Daher, 2019a).

Asimismo, durante el 2000 y 2010 la economía siria se caracterizó por la liberalización del comercio exterior. Con ello, se llevaron a cabo sucesivas adaptaciones legislativas con el fin de atraer a corto plazo a las inversiones procedentes de países del golfo. Esta serie de reformas económicas facilitaron el surgimiento grandes compañías, dedicadas especialmente al sector de la construcción o a la industria de servicios, cuyos dueños y altos cargos mantenían un vínculo muy cercano al presidente. El caso más representativo es el de Rami Makhlouf, primo de Bashar Al Asad, cuyo imperio incluye compañías de telecomunicaciones, gas y construcción, bancos, y aerolíneas, entre otros. Es así como se generó lo que en inglés algunos académicos denominan "Crony Capitalism" para definir la situación económica post-2000 en Siria. El economista y académico suizo- sirio Joseph Daher señala que este fenómeno se produce cuando los propietarios de grandes negocios explotan sus vínculos con el Estado y el gobierno como medio para obtener ventaja frente a sus competidores (Daher, 2019b).

Podemos afirmar que la naturaleza neopatrimonial del régimen sirio ha favorecido a la emergencia de este proceso de privatización mafiosa, que ha sido cultivado durante décadas mediante la concesión de privilegios al círculo cercano al régimen y la oferta de oportunidades únicamente a quienes hubieran demostrado su lealtad a él, o suponían un elemento de apoyo para la consolidación de su poder. Mientras la riqueza generada de este proceso era retenida por esta pequeña oligarquía, la calidad de los bienes y servicios básicos decaía y las clase media y obrera (urbana y rural) quedaban excluidas los beneficios resultantes de esta nueva economía, cuyos negocios quedaron concentrados en la capital y en grandes ciudades.

Es por ello que entre las consecuencias más graves de estas reformas neoliberales nos podemos encontrar: el aumento del precio de la vivienda y de los alimentos básicos, la decadencia del sector público y agrícola, la adaptación de la legislación con el fin de favorecer a la inversión en terrenos y construcción de edificios de lujo mientras se generaban impedimentos para llevar a cabo transacciones inmobiliarias fronterizas (Yazigi, 2017). Una de las consecuencias más graves de esta situación fue la migración masiva de la población desde las zonas rurales a los suburbios o cinturones de las grandes ciudades, que causó el aumento de las infraviviendas y la generación de una tensión social agravada por las malas condiciones de vida y la ausencia de mecanismos que aseguraran una justicia social. 
Mientras que los esfuerzos de Hafez Al Asad fueron invertidos en mantener una estructura que asegurara la coordinación entre el nivel central y local, y en cierto grado fomentara la cooperación entre los actores locales, las reformas económicas emprendidas por Bashar Al Asad y con ellas el surgimiento una nueva élite clientelista tuvieron como resultado en lo que al ámbito local se refiere, en el tratamiento de las localidades como proyectos empresariales y espacios de implementación de agendas personales. Prueba de ello es que a día de hoy, Siria ocupa el segundo lugar del ranking de países corruptos elaborado por Transparency International ${ }^{11}$.

\section{Siria post-2011: Nuevos actores en el escenario de conflicto ¿una oportunidad para un nuevo orden socio-político?}

A menudo se ha categorizado a Siria como Estado fallido (Lynch, 2016; Betz, 2019) o frágil (Werrell et al, 2015; Perthes, 2019) debido a la disfuncionalidad de sus instituciones. Asimismo, algunos autores como Raymond Hinnebusch han dedicado esfuerzo a analizar el proceso de deconstrucción del Estado sirio. Sin embargo, y teniendo en cuenta lo anteriormente analizado, podemos coincidir con Steven Heydmann, ya que da un paso más y define a Siria como un "Estado feroz". Esta denominación se basa en una cuestión clave para entender la arquitectura del régimen de Asad, que ha convertido a Siria en un Estado donde la prioridad de las élites dominantes es su propia supervivencia y que además, cuenta con el apoyo de instituciones diseñadas expresamente para la consecución de este objetivo (Heydmann, 2018: 6). No obstante, cabe preguntarse si este modelo de subsistencia ha sobrevivido al estallido del conflicto, o si a cambio, el propio conflicto se presenta como una nueva oportunidad para la articulación de un nuevo orden socio-político.

Para Steven Heydmann la conclusión general sobre la dirección que ha de tomar la reconstrucción del conflicto sirio se basa en una cadena de causa-efecto: la violencia del conflicto es producto de la fragilidad estatal, a su vez, la violencia ha causado la desintegración de las instituciones establecidas en el pre-conflicto, lo que induce a la apertura de un espacio de reconstrucción que termine con las condiciones que retroalimentan la debilidad y la destrucción, en el que operan nuevos actores emergidos en el propio conflicto, dispuestos a liderar la transformación del orden pre-establecido. (Heydmann, 2018: 7). Sin embargo, estos nuevos actores emergidos en el marco del conflicto han de coexistir con los preexistentes dentro en una dinámica caracterizada por la competición y la colaboración. Debemos tener en cuenta que, a causa de la desintegración de las instituciones estatales, diferentes actores no estatales (consejos locales, organizaciones de sociedad civil, grupos terroristas, tribunales de la sharía, etc.) han asumido las funciones que hasta entonces ejercía el Estado: proveer servicios básicos y protección, suministro de ayuda humanitaria, suministro de bienes, seguridad, mediación, control del territorio, resolución de disputas, etc. Ya sea con el fin de preservar sus antiguos privilegios en el caso de los actores tradicionales (lideres de familias notables, lideres tribales, líderes religiosos, empresarios y hombres de influencia), o por el mantenimiento de su actividad en el caso de los nuevos, la efectividad de sus acciones y las dinámicas del conflicto les ha permitido obrar de una manera más flexible, permitiéndose establecer relaciones de dependencia no solo con el régimen, sino también con otros actores implicados en el conflicto.

Ante la imposibilidad de analizar todos estos actores locales (contrabandistas, hombres de negocios beneficiados por la guerra, grupos paramilitares, líderes religiosos, líderes de tribus, terratenientes,

11 Se puede consultar la base de datos completa, con mapa interactivo incluido en el siguiente enlace: https://www.transparency.org/en/cpi [consulta: 18/12/2020]. 
N. Al-Hussen Villa, Poder y dependencia: Los intermediarios locales...

etc.) hemos elegido dos muy diferentes entre sí, y que representan la coexistencia de intermediarios pertenecientes a la estructura pre-2011 y la nueva: las tribus y los nuevos líderes locales.

\section{La decadencia de los jefes tribales como elemento de contención}

Pese a la extensión de la idea de que el tribalismo ha supuesto una forma de organización sociopolítica, y un poderoso instrumento de movilización, como fue el caso de la organización de las primeras protestas en Hasakah en febrero de 2011 (Heras y O'Leary, 2013) no podemos catalogar como homogénea su capacidad de influencia ni tampoco su posicionamiento durante el conflicto. Por una parte, el concepto de solidaridad compartida por el que se rige su acción colectiva ha motivado a que un gran número de tribus se posicionaran a favor de las protestas organizadas en el 2011 y rechazaran la represión ejercida por parte del régimen. Por ejemplo, la ciudad de Daraa las tribus impulsaron la organización de las primeras protestas en la localidad a causa de la indignación despertada tras la tortura de dos menores detenidos por las fuerzas de seguridad por la realización de grafittis en contra del régimen (Abouzeid, 2011).

Algunos líderes tribales intentaron mediar la liberación de los menores sin éxito. La extrema violencia que sufrieron estos menores por parte de las fuerzas de seguridad violó el acuerdo implícito y pre-existente en cuanto al mecanismo informal de resolución de disputas (sulh), una competencia exclusiva de las familias y tribus con influencia (Al-Jabassini y Ezzi, 2021), en la que solo se requería la presencia de los agentes de seguridad en calidad de testigo o elemento coercitivo, y cuya intervención era necesaria únicamente en casos extremos. La detención, tortura y mutilación de los menores de Daraa, supuso la ruptura de este acuerdo que constituía la base de la vieja estructura de poder. Asimismo, la incapacidad de los líderes tribales progubernamentales de contener las protestas demostró la debilitación de su autoridad local. De hecho, con la escalada de represión y violencia, el rol que venían ejerciendo durante décadas como intermediarios perdía gradualmente su efectividad (Dukhan, 2014: 6-7).

Conscientes de la capacidad de influencia de las tribus, y el peligro que suponía la pérdida de su lealtad, el régimen sirio organizó varias conferencias tribales que fueron promocionadas por varios medios, con el fin de reunir a los sheikhs ${ }^{12}$ y líderes más influyentes, obligándoles así a demostrar públicamente la su hacia Bashar Al Asad. El acontecimiento más significativo fue la visita del propio presidente en junio de 2011 a Raqqa ${ }^{13}$ (Al-Musarea, 2019) con el objetivo de solicitar el apoyo de los líderes de las tribus. No obstante, al menos en el caso de Raqqa los esfuerzos resultaron en vano, ya que meses después se proclamaría como la primera capital de provincia liberada del régimen.

Tras la visita de Al Asad, varios actores comenzaron a ser conscientes de la importancia que suponía el establecimiento de una alianza con las tribus. Es así como se financiaron varios grupos armados que defendían los intereses de los clanes y las tribus, así como los de sus mecenas regionales o internacionales. Podemos afirmar que la retirada del régimen en las zonas tribales, principalmente

\footnotetext{
12 Título árabe designado a líderes religiosos principalmente, aunque también puede ser aplicable en un contexto local a personalidades de relevancia política o social.

13 históricamente marginada por el gobierno central.
} 
en el noroeste sirio, impulsó la creación de un nuevo escenario sin precedentes en el que diversos grupos radicales, así como otros actores implicados intentaron explotar e instrumentalizar las divisiones tribales, como fue el caso de Daesh. En este caso, una de las primeras acciones del grupo al tomar el control de Raqqa fue la apertura de una oficina de Relaciones Públicas, cuyo objetivo era regular los asuntos tribales. Entre las funciones principales destacaba el reclutamiento de guerreros, así como apoyar, orientar y adecuar los mensajes públicos y acciones del grupo yihadista.

Tras la liberación de la ciudad del grupo yihadista por parte de las FDS (Fuerzas Democráticas Sirias) y con el fin de legitimar su gobierno local y buscar la aceptación de la población, mayoritariamente árabe, las fuerzas kurdas también intentaron absorber a las tribus dentro de sus estructuras. Al mismo tiempo, los esfuerzos del régimen en reconquistar el apoyo de las tribus se vieron reflejados en la reunión celebrada en enero de 2019 en Ithryiah (a $125 \mathrm{~km}$ de Raqqa), en la que se convocaron a más de un centenar de jefes de tribus (Al-Musarea, 2019).

En definitiva, en un primer momento el vacío de poder y el aislamiento generado por el propio conflicto, reforzó la identidad tribal parcialmente o al menos en lo que a seguridad y a mecanismos tradicionales de resolución de disputas se refiere. No obstante, y con el transcurso del conflicto, las dinámicas tribales fueron erosionadas debido a la emergencia y consolidación de otros actores que podían contribuir de una manera más eficaz a la supervivencia y protección de las comunidades locales. Asimismo, la sumisión de los jefes tribales al régimen durante décadas, así como su ambigüedad y flexibilidad en su posicionamiento con los diferentes bandos implicados a lo largo del conflicto se convirtieron en motivos de peso para que su autoridad y credibilidad se pusieran en entredicho entre los propios miembros de sus tribus. Es por ello que aunque el sistema tribal permanezca en Siria, sus líderes contarán únicamente con una autoridad simbólica, y su labor quedará delimitada a labores de reconciliación y estabilización de las áreas donde habitan sus tribus (Khaddour, 2017).

\section{El auge de los jóvenes líderes locales}

Los jóvenes fueron protagonistas indiscutibles del levantamiento popular sirio, y en general de la denominada "Primavera árabe". La mayoría de ellos eran menores de 35 años, afectados por las consecuencias de las reformas económicas emprendidas, entre ellas podemos mencionar el paro y la desigualdad de oportunidades (Bonfatti, 2017; Daher, 2019a).

En un primer lugar, estos jóvenes demostraron una gran habilidad para organizar las primeras protestas y burlar las prohibiciones impuestas por la dictadura. El excelente uso de la tecnología y de las redes sociales agilizó el proceso de convocar las diferentes manifestaciones que se iban extendiendo a lo largo del territorio sirio. Una vez comenzada la escalada de violencia, los jóvenes activistas recurrieron a esta habilidad para documentar las violaciones de derechos humanos que estaban sufriendo los manifestantes a manos de las fuerzas del régimen. Posteriormente, esta actividad inicialmente espontánea e informal fue institucionalizada con la creación de los Comités de Coordinación local (Carnegie Middle East Center, 2012), cuyas funciones principales se basaban en la llamada a la desobediencia civil, la documentación de violaciones de derechos humanos, y la asistencia a la población en sus necesidades más inmediatas. De esta manera, los activistas no solo ejercieron un rol de mediadores entre sus comunidades y los movilizadores, sino también entre las comunidades locales y otros actores externos que comenzaban a ser conscientes, gracias a la labor de estos jóvenes, de la crisis que atravesaba el país levantino (Khalaf et al, 2015). 
Una vez comenzado el proceso de implosión de las estructuras estatales, las funciones de los comités ya no resultaban suficientes para abordar este nuevo escenario repleto de cambios y retos. La liberación de localidades exigía nuevas responsabilidades como es la gestión del territorio y de los servicios básicos, así como la protección de la población y la mediación en asuntos de vital importancia como puede ser la asistencia humanitaria. Surgen así pequeñas estructuras administrativas denominadas como Consejos Locales, cuya función principal es asumir las funciones que hasta entonces eran competencia del estado central. El ideario de este laboratorio de autogestión conformado en las zonas liberadas por las fuerzas opositoras fue el anarquista Omar Aziz. Para este activista, la acción revolucionaria no podía completarse sin alcanzar la autonomía real del Estado, es decir, no bastaba con sublevarse y sumarse a la acción de protesta, sino que también era necesario sustituir directamente al Estado, especialmente en aquellas acciones que afectan a la vida cotidiana de las personas (Aziz, 2013).

No obstante, estas células de gestión local se enfrentaron a múltiples dificultades relacionadas con la propia naturaleza del conflicto. La ausencia de un liderazgo político efectivo en la oposición siria, además de su caótica organización y sus múltiples divisiones, junto al extremo ejercicio de violencia en las zonas liberadas acentuó la dimensión geográficamente localizada del conflicto. Por este motivo, en vez de obrar de manera coordinada con sus similares en otras partes del territorio, los diferentes consejos locales actuaron en base a una lógica individual, anteponiendo su propia supervivencia y la de su comunidad local. Se trata de una acción instintiva enmarcada dentro de lo que el intelectual sirio denomina "Estado de la naturaleza" (Haj Salleh, 2018: 91- 104). Para este disidente e intelectual sirio, se trata de una noción colectiva de supervivencia causada por el caos del conflicto, que evita basar las acciones en una estrategia racional y nacional, ya que prioriza la búsqueda de soluciones que afectan al ámbito más próximo (es decir, el local) así como el cálculo de resultados a corto plazo en un contexto extremo como es el de un conflicto armado y que se encuentra en constante dinámica.

Aun así, la sombra del viejo orden acechó a estas nuevas estructuras. Pese a que existe un acuerdo general entre analistas y académicos entorno a la efectividad de estas estructuras locales y a su alto grado de nivel democrático debido a sus mecanismos de elección, transparencia y fomento del debate y consulta pública (Maalouf et al, 2014; Hajjar et al, 2017), los consejos locales han sido acusados de limitar el acceso a puestos de responsabilidad imponiendo criterios específicos que eximen a la mayoría de la población. Un informe Swiss Peace señala como en algunos casos los mecanismos de selección de sus miembros no han sido transparentes, o han estado influenciados por la recomendación o sugerencia de familias locales de gran poder.

La caótica canalización de la financiación por parte de donantes internacionales también ayudó a la monopolización del poder y el acceso a los recursos por parte de perfiles determinados. Podemos señalar dos cuestiones claves que justifican este fracaso en la asistencia a Siria: La falta de una estrategia común de financiación y una lógica institucionalizadora enmarcada en la concepción de una paz neoliberal, en la que se prima la burocratización y la reconstrucción de las instituciones políticas en base a los estándares occidentales frente a la adecuación de medidas al contexto local específico, lo que implica por ejemplo la atención a las relaciones informales y fluidas establecidas en el conflicto o la regeneración de la confianza mutua, o del tejido social (Khalaf, 2015). 
Por ejemplo, las exigencias de las convocatorias de financiación, así como la asistencia a cursos y programas de capacitación técnica posibilitan únicamente el acceso a esos puestos a personas con conocimiento de lengua inglesa, dispuesta a realizar viajes para atender a cursos, así como a adecuar las acciones a implementar en consonancia con la agenda de los donantes, en vez de a las necesidades reales existentes (Swehat, 2017). Es por ello que podemos observar que el acceso a recursos, así como la concentración de poder queda delimitada a perfiles específicos: clase media con estudios, habitantes de áreas metropolitanas y con conocimiento de inglés (Khalaf, 2015: 5254).

Podemos señalar que del mismo modo que el régimen llevó a cabo su estrategia de cooptación de las tribus en los territorios recuperados, los consejos locales también constituyeron un socio necesario para la reconsolidación del régimen el escenario de posconflicto, ya que, además, el Decreto Legislativo 107 le concede cierto grado de autonomía a los consejos locales sobre algunos aspectos económicos y relacionados con el ámbito de la construcción. El éxito del plan de absorción de los consejos por parte del régimen con el fin de restaurar su modelo de control político a nivel local se ve reflejado en los resultados de las últimas elecciones municipales llevadas a cabo en septiembre de 2018 en los territorios ya bajo el control del régimen, en el que el $70 \%$ de los candidatos pertenecían al partido de Al Baaz (Youssef, 2019: 72-72).

\section{Conclusiones}

El control de las localidades ejercido por parte de las autoridades centrales en Siria ha sido posible, incluso antes de su constitución como Estado moderno, gracias a la labor de intermediarios que han ejercido de nexo entre el gobierno central y la población local. Estos intermediarios, debido a su status y capacidad de influencia dentro de sus comunidades locales se presentaron como un socio necesario para la extensión del dominio de las autoridades centrales en las áreas periféricas del país, así como su colaboración en la contención de posibles sublevaciones. Hemos visto como la relación centro - periferia durante el imperio otomano, fue asumida por los llamados "ayan", y que de alguna manera mantuvieron un rol similar en el mandato francés.

Esta relación de dependencia basada en la lealtad transaccional, es decir en la sumisión a cambio de privilegios e incentivos, se ha ido manteniendo a lo largo de las diferentes etapas y mandatos en la Siria contemporánea, lo que demuestra su resiliencia y su conformación como componente esencial para supervivencia del régimen. Es así como podemos diferenciar entre dos tipos intermediarios: los tradicionales (cuya influencia y autoridad emana de su posición en el pasado: cabezas de familias de renombre, jefes de tribus, comerciantes importantes, etc.) y los nuevos, creados por el propio baazismo en su primera década del poder en base a corporativismo autoritario. Mientras que Hafez Al Asad logró absorber a ambos grupos dentro de las estructuras del Estado creando cierto grado de cooperación entre ambas, su hijo Bashar Al Asad impulsó la creación de una nueva élite amparada bajo el paraguas del capitalismo clientelista generado a partir de las medidas económicas emprendidas tras su llegada al poder.

La organización del levantamiento popular, y posteriormente el estallido del conflicto originaron nuevos intermediarios y líderes locales, lo que supuso la creación de un espacio de coexistencia entre estos y sus predecesores. La indiscriminada violencia ejercida, la segmentación caótica de la oposición y su carencia de un liderazgo efectivo, junto la incapacidad del Estado central de responder a las necesidades primarias y de proveer de servicios básicos a los ciudadanos permitieron que estos asumieran parte de las responsabilidades principales del Estado. Esto incluye la gestión de los servicios básicos, la resolución de disputas y la mediación con otros actores 
implicados en el conflicto. A pesar de continuar manteniendo una naturaleza dependiente, con la desintegración de las instituciones del estado, y la implicación de otros actores estatales y no estatales en el conflicto, los intermediarios han explotado su efectividad a hora de asumir responsabilidades sobre el terreno, por lo que han manifestado una mayor flexibilidad y autonomía a la hora de establecer relaciones de dependencia con diferentes actores, no solo con el régimen tal y como requería el orden pre 2011.

A pesar de existir un consenso general entorno a la oportunidad que ha supuesto el conflicto para la creación de nuevos actores que lideraran una transformación profunda, que superara las deficiencias del orden sociopolítico sirio anterior la guerra, lo cierto es que lo analizado muestra lo contrario. A pesar del nacimiento de nuevos actores que en un primer momento han supuesto una oportunidad para la ruptura con las prácticas clientelistas del pasado, lo cierto es que las condiciones y características del conflicto en su nivel local han facilitado la reaparición de estas dinámicas, tanto por parte de los intermediarios tradicionales como los emergidos en el marco del conflicto. Podemos afirmar que esta cultura clientelista, sistemáticamente reproducida durante décadas ha quedado arraigada en la noción del sistema de control y distribución de poder, hasta el punto de que ni el alto grado de violencia ha podido destruirla. Asimismo, la intervención de actores externos en el conflicto y su búsqueda de socios para la implantación de su agenda según sus intereses y sus estrategias para la consecución de una paz neoliberal también ha contribuido a la creación de nuevas formas de dependencia y a la concentración del poder y el acceso a los recursos en manos de una minoría.

Entre las limitaciones de este trabajo se encuentra la decisión de haber analizado el comportamiento y el recorrido de únicamente dos actores muy específicos y de diferente naturaleza. Para futuras investigaciones sería interesante estudiar las dinámicas de otros tipos de intermediarios y establecer una correlación entre sus principales rasgos, con el fin de extrapolar una conclusión más generalizable.

La victoria de Bashar y su consolidación en el poder en el escenario de posconflicto supone una nueva fase del ciclo de las relaciones de dependencia centro-periferia preestablecidos. Con la reconquista de los territorios previamente controlados por otras partes del conflicto y la cooptación de sus élites que ayudará a que sus intereses sean asegurados en los planes de reconstrucción, el régimen consolida su poder y reanuda su estrategia de control político a través de estas redes, transformadas y regeneradas, pero que al fin y al cabo continúan conservando su naturaleza dependiente en busca de su propio beneficio.

\section{Bibliografía}

ABOUZEID, Rania: "Syria's Revolt: How Graffiti Stirred an Uprising", Time, 22 de marzo de 2011, disponible en http://content.time.com/world/article/0,8599,2060788,00.html [consulta: 20 de mayo de 2021]

AL-JABASSINI, Abdullah y EZZI, Mazen: "Tribal 'Sulh' and the Politics of Persuasion in Volatile Southern Syria", European University Institute, 22 de marzo de 2021, disponible en 
https://cadmus.eui.eu/bitstream/handle/1814/70657/QM-02-21-370-EN-N.pdf?sequence=1 [consulta: 29 de septiembre de 2021]

AL-MUSAREA, Ammar: "The Role of Syrian Tribes Betting on a Lost Cause", Washington Institute, 26 de marzo de 2019, disponible en https://www.washingtoninstitute.org/policy-analysis/rolesyrian-tribes-betting-lost-cause [consulta: 28 de enero de 2021]

ÁLVAREZ-OSSORIO, Ignacio (2016): Siria: Revolución, sectarismo y yihad, Madrid, La Catarata.

ÁLVAREZ-OSSORIO, Ignacio (2011): “Las paradojas del islam político en Siria", Revista CIDOB d' Afers Internacionals, no 93, pp. 163-178, https://raco.cat/index.php/RevistaCIDOB/article/view/240764 [consulta: 17 de septiembre de 2021]

AZIZ, Omar (2013): A Discussion Paper on Local Councils in Syria. The Anarchist Library

BATATU, Hanna (1999): Syria's peasantry, the descendants of its lesser rural notables, and their politics, Nueva Jersey, Princeton University Press.

BALANCHE, Fabrice (2009): "Clientelism, Communitarianism, and Territorial Fragmentation in Syria", A contrario, vol. 11, no 1, pp. 122-150. DOI: 10.3917/aco.061.0122

BECKER, Carmen (2006): "Strategies of Power Consolidation in Syria Under Bashar al-Asad: Modernizing Control Over Resources", The Arab Studies Journal, vol. 14, no 1, pp. 65-91.

BETZ, Frederick (2019): "Political Theory of Societal Association: Case of the Failed State of Syria, Part 1", Open Journal of Social Sciences, 7, pp. 271-296. DOI: 10.4236/jss.2019.711020

BONFATTI, Alice: "The Socio-Economic Roots of Syria's Uprisin". Al Jumhuriya, 21 de septiembre de 2017, disponible en https://www.aljumhuriya.net/en/content/socio-economic-roots-syria'suprising [consulta: 3 de febrero de 2020]

CARNEGIE MIDDLE EAST CENTER (2012): "Local Coordination Committees of Syria“, Carnegie Middle East Center, 20 de diciembre de 2012, disponible en https://carnegiemec.org/diwan/50426?lang=en [consulta: 5 de febrero de 2021]

CHATTY, Dawn (2010): "The Bedouin in contemporary Syria: the persistence of tribal authority and control", The Middle East Journal, vol. 64, no 1, pp. 29-49. DOI: 10.3751/64.1.12

DAHER, Joseph (2019a): "The political economic context of Syria's reconstruction: a prospective in light of a legacy of unequal development", Robert Schuman Centre for Advanced Studies, 13 de marzo de 2019, disponible en https://op.europa.eu/en/publication-detail/-/publication/a48ab131460e-11e9-a8ed-01aa75ed71a1 [consulta: 28 de marzo de 2021]

DAHER, Joseph (2019b): "The Paradox of Syria's Reconstruction", Carnegie Middle East Center, 4 de septiembre de 2019, disponible en https://carnegie-mec.org/2019/09/04/paradox-of-syria-sreconstruction-pub-79773 [consulta: 18 de marzo de 2021]

DAM, Nikolaos van (1979): The Struggle for Power in Syria: Politics and Society Under Asad and the Ba'th Party, Londres, I. B. Tauris.

DERDERIAN, Dzovinar; SIPAHI, Ali y TOLGA CORA, Yasar (2016): The Ottoman east in the nineteenth century, Londres, I. B. Tauris.

DIWAN, Ishac; MALIK, Adeel y ATIYAS, Izak (2019): Crony Capitalism in the Middle East: Business and Politics from Liberalization to the Arab Spring, Oxford, Oxford University Press.

DUKHAN, Haian (2019): State and Tribes in Syria: Informal Alliances and Conflict Patterns, Londres, Routledge.

DUKHAN, Haian (2014): "Tribes and tribalism in the Syrian uprising", Syria Studies, vol. 6, no 2, pp. 1-28. 
N. Al-Hussen Villa, Poder y dependencia: Los intermediarios locales...

EMRENCE, Cem (2008): "Imperial paths, big comparisons: the late Ottoman Empire", Journal of Global History, vol. 3, no 3, pp. 289-311. DOI: 10.1017/S1740022808002738

GELVIN, James (2006): “The Politics of Notables' Forty Years After", Middle East Studies Association Bulletin, vol. 40, no 1, pp. 19-29.

HAJ SALLEH, Yassin (2018): Siria. La revolución imposible, Madrid, Oriente y Mediterráneo.

HAJJAR, Bahjat; VON BURG, Hilal; SANTASCHI, Martina; GHARIBAH, Mazen y SHARABAJI, Mazhar: "Perceptions of governance- The experience of local administrative councils in opposition-held Syria", SwissPeace, marzo de 2017, disponible en https://www.swisspeace.ch/publications/reports/perceptions-of-governance-the-experience-oflocal-administrative-councils-in-opposition-held-syria [consulta: 5 de febrero de 2021]

HERAS, Nicolas y O'LEARY, Carole (2013): “The Tribal Factor in Syria's Rebellion: A Survey of Armed Tribal Groups in Syria", Jamestown Foundation, 27 de junio de 2013, disponible en: https://jamestown.org/program/the-tribal-factor-in-syrias-rebellion-a-survey-of-armed-tribalgroups-in-syria/ [consulta: 13 de octubre de 2020]

HEYDMANN, Steven: "Beyond fragility: Syria and the challenges of reconstruction in fierce states", Foreign Policy at Brookings: The New Geopolitics: Middle East, 1 de junio de 2018, disponible en https://www.brookings.edu/research/beyond-fragility-syria-and-the-challenges-ofreconstruction-in-fierce-states/ [consulta: 19 de marzo de 2021]

HINNEBUSCH, Raymond A. (1976): "Local politics in Syria: organization and mobilization in four village cases", Middle East Journal, vol. 30, no 1, pp. 1-24.

HOURANI, Albert (1981): The emergence of the modern Middle East, California, University of California Press.

HOURANI, Albert (1968): "The Politics of Notables" en POLK, William y CHAMBERS, Richard: Beginnings of modernization in the Middle East: The nineteenth century, Chicago, Publications of the Middle Eastern Journal University of Chicago.

HUMAN RIGHTS WATCH (HRW) (2019): “World Report 2019: Syria Events of 2018”, HRW, disponible en https://www.hrw.org/world-report/2019/country-chapters/syria [consulta: 18 de noviembre de 2020]

INTERNATIONAL CRISIS GROUP (2004): "Syria Under Bashar (II): Domestic Policy Challenges", ICG Middle East Report, ํㅡ 24.

INALCIK, Halil (1976): "The rise of the Ottoman Empire" en Cook, M.A. (1976): A History of the Ottoman Empire to 1730, Cambridge, Cambridge University Press, pp. 10-53.

KHADOUR, Khedder (2017): "Local wars and the chance for descentralized Peace in Syria". Carnegie Middle East, marzo de 2017, disponible en https://carnegie-mec.org/2017/03/28/local-wars-andchance-for-decentralized-peace-in-syria-pub-68369 [consulta: 13 de noviembre de 2020]

KHADOUR, Khedder y MAZUR, Kevin (2019): “Local Intermediaries in post-2011 Syria. Transformation and Continuity", Friederich-Ebert-Stiftung, disponible en http://library.fes.de/pdffiles/bueros/beirut/15547.pdf [consulta: 07 de octubre de 2020]

KHALAF, Rana; RAMADAN, Oula y STOLLIES, Friederieke (2015): Activism in Difficult Times - Civil Society Groups in Syria (2011-2014), Beirut, Fundación Friedrich Ebert/Badael. 
KHALAF, Rana: "Government without governance in Syria", CORE, 16 de junio de 2015, disponible en https://ojs.st-andrews.ac.uk/index.php/syria/article/view/1176 [consulta: 14 de enero de 2021]

KHOURY, Philip (1991): "Continuity and change in Syrian political life: the nineteenth and twentieth centuries", The American Historical Review, pp. 1374-1395. DOI: 10.2307/2165276

LAPIDUS, Ira (1967): Muslim Cities in the Later Middle Ages, Cambridge, Cambridge University Press.

LYNCH, Marc (2016): "Failed States and Ungoverned Spaces", The ANNALS of the American Academy of Political and Social Science, vol. 668, no 1, pp. 24-35. DOI: $10.1177 / 0002716216666028$

MAALOUF, Marwan; SAWAH, Wael y ZIADEH, Radwan (2014): "Local Governance inside Syria", Refworld, marzo de 2014, disponible en: https://www.refworld.org/pdfid/5416e7d14.pdf [consulta: 9 de noviembre de 2020]

PIERRET, Thomas (2009): "Sunni Clergy Politics in the Cities of Ba'thi Syria," en: LAWSON, Fred (dir.): Demystifying Syria, Londres, Saqi, pp. 70-84.

PERTHES, Volker: "Syria: too fragile to ignore; military outcomes, external influence and European options", SWP German Institute for International and Security Affairs, 7 de febrero de 2019, disponible en https://www.swp-berlin.org/10.18449/2019C07/ [consulta: 19 de marzo de 2021]

QUATAERT, Donald (2005): The Ottoman Empire, 1700-1922, Cambridge, Cambridge University Press.

ROGAN, Eugene (2010): Los árabes, Barcelona, Crítica.

RUIZ DE ELVIRA, Laura: “¿Por qué luchan los sirios?", Ethic, 4 de mayo de 2012, disponible en https://ethic.es/2012/05/por-que-luchan-los-sirios/ [consulta: 1 de febrero de 2021]

RUIZ DE ELVIRA, Laura y ZINTL, Tina (2014): "The end of the Ba'athist social contract in Bashar AlAsad's Syria: Reading sociopolitical Transformations through charities and broader benevolent activism", International Journal of Middle East Studies, pp. 329-349. DOI:10.1017/S0020743814000130

RUIZ DE ELVIRA, Laura; SCHWARZ, Christoph y WEIPERT-FENNER, Irene (eds.) (2018): Clientelism and patronage in the Middle East and North Africa: Networks of dependency, Londres, Routledge.

SALZMANN, Ariel (2004): Tocqueville in the Ottoman Empire: rival paths to the modern state, Leiden, Brill.

SHAW, Stanford J. y SHAW, Ezel Kural (1977): History of the Ottoman Empire and Modern Turkey: Volume 2, Reform, Revolution, and Republic: The Rise of Modern Turkey 1808-1975, Cambridge, Cambridge University Press.

SWEHAT, Yassin: "Syrian civil society organizations and the obsession with management". Ennab Baladi, 29 de julio de 2017, disponible en https://english.enabbaladi.net/archives/2017/07/syriancivil-society-organizations-obsession-management/\#ixzz6IXJuKQSO [consulta: 3 de enero de 2021]

UNITED NATIONS OFFICE OF HUMANITARIAN AFFAIRS (UNOCHA) (2019): "Syria: UN remains concerned over hostilities in north-west", UNOCHA, disponible en https://www.unocha.org/story/syria-un-remains-concerned-over-hostilities-north-west [consulta: 18 de noviembre de 2020]

WERRELL, Caitlin E.; FEMIA, Francesco y STERNBERG, Troy (2015): "Did we see it Coming?: state fragility, climate vulnerability, and the uprisings in Syria and Egypt", SAIS Review of International Affairs, vol. 35, no 1, pp. 29-46. DOI: 10.1353/sais.2015.0002

WIMMEN, Heiko (2016): "Syria's Path from Civic Uprising to Civil War", Carnegie Endowment for International Peace, 22 de noviembre de 2016, disponible en 
https://carnegieendowment.org/2016/11/22/syria-s-path-from-civic-uprising-to-civil-war-pub66171 [consulta: 29 de marzo de 2021]

YAZIGI, Yihad (2017): "Destruct to Reconstruct. How the Syrian Regime Capitalises on Property Destructiond and Land Legislation", Friederich-Ebert-Stiftung, disponible en: http://library.fes.de/pdf-files/iez/13562.pdf [consulta: 18 de noviembre de 2020]

YOUSSEF, Maryam:" Syrian people suffer after Pyrrhic victory", Blog LSE, 12 de febrero de 2019, disponible en https://blogs.Ise.ac.uk/crp/2019/02/12/syrian-people-suffer-after-pyrrhic-victory/ [consulta: 28 de marzo de 2021] 